\title{
Role of Fibroscan for early detection of hepatocellular carcinoma (HCC) in hepatitis $C$ cirrhotic patients
}

\author{
Ahmed Elhosieny Ebrahim', Mona Ahmed Helmy Shehata², Sabry Abou-saif ${ }^{2}$, Manal fathy Hamisa ${ }^{3}$, \\ Sherief Abd-Elsalam ${ }^{2 *}$ and Mohamed Yousef ${ }^{2}$
}

\begin{abstract}
Background: Liver cancer (HCC) ranks as the fifth most prevalent cancer worldwide and is ranked third as a common cause of death due to cancer. The benefit of Fibroscan in assessing the risk of developing liver cancer that was evaluated in this work was not fully demonstrated. The aim of this work was to study the role and clinical significance of Fibroscan for early detection of liver cancer in hepatitis C patients with cirrhosis.

Results: As regards binary logistic regression for predictors of HCC, it was found that Child C, AST, Fibroscan, and AFP were predictors for developing HCC. Liver stiffness values were significantly high in all groups, and the specific cutoff value for hepatocellular carcinoma detection was above $24 \mathrm{kPa}$ in hepatitis $\mathrm{C}$ virus patients. Therefore, liver stiffness of more than $24 \mathrm{kPa}$ can be considered as an independent risk factor for the development of liver cancer in HCV patients.
\end{abstract}

Conclusion: Liver stiffness of more than $24 \mathrm{kPa}$ was an independent risk factor for developing new liver cancer in HCV patients.

Keywords: HCC, Fibroscan, Liver disease, Cirrhosis, Prediction, Prevalence

\section{Background}

Liver cancer $(\mathrm{HCC})$ ranks as the fifth most prevalent cancer worldwide, and is ranked third as a common cause of death due to cancer $[1,2]$. In Egypt, the prevalence of hepatocellular carcinoma increased markedly in the last decade due to the high prevalence of hepatitis $\mathrm{C}$ virus and the improved survival for cirrhotic patients allowing time for some of them to develop HCC [3-8].

For improvement in the fate of liver cancer, adequate treatment after early detection is important. To this end, it is critical to identify high-risk groups for liver cancer and to conduct appropriate screening in the clinical practice of chronic liver disease $[9,10]$.

\footnotetext{
* Correspondence: sherif_tropical@yahoo.com

${ }^{2}$ Department of Tropical Medicine, Faculty of Medicine, Tanta University Hospital, Tanta University, El Geish Street, Tanta, Gharbia Governorate, Egypt Full list of author information is available at the end of the article
}

It has been postulated that hepatitis virus infection, old age, male sex, liver cirrhosis alanine aminotransferase (ALT) elevation, low albumin (Alb), low platelets (Plt), and $\alpha$-fetoprotein (AFP) elevation are risk factors for HCC; however, liver cirrhosis is the most important risk factor irrespective of its etiology [11, 12].

Liver cirrhosis has been evaluated by liver biopsy, as the histology is the gold standard for quantitative fibrosis assessment; but liver biopsy is associated with several problems such as invasiveness, sampling errors, and diagnostic differences between pathologists. This makes it unpopular among patients and impractical for serial assessments of patients with chronic liver disease. With the development of Fibroscan using transient elastography, it became possible to estimate the elasticity of the liver $[13,14]$. An accurate quantification of the degree of liver fibrosis is necessary for prognosis and guiding surveillance [15]. 
The accuracy of Fibroscan diagnosis of cirrhosis has been widely recognized in many chronic liver diseases except for some liver conditions such as congestion, severe infections, or cholestasis, which may be overestimating cirrhosis with Fibroscan [16].

Recently, the risk of liver cancer was assessed based on liver stiffness measured by Fibroscan among the European population [17]; however, in most reports, the risk of liver cancer has been indirectly assessed based on the value of cirrhosis as measured by Fibroscan; however, HCCassociated liver stiffness was not directly assessed [18].

Additionally, the effectiveness of Fibroscan in predicting the risk of HCC has not been fully elucidated that is assessed in this work. The aim of this work was to study the role and clinical significance of Fibroscan for early detection of HCC in hepatitis $\mathrm{C}$ cirrhotic patients.

\section{Methods}

This was a cross-sectional study included fifty (50) HCV patients with liver cirrhosis selected from a major university hospital. Approval of the study was obtained by the institutional ethical committee. They were divided into two groups as the following: group I: included 25 $\mathrm{HCV}$ cirrhotic patients with HCC and group II: included $25 \mathrm{HCV}$ cirrhotic patients without HCC.

Inclusion criteria: adult HCV cirrhotic patients above 18 years, with or without hepatocellular carcinoma (HCC)

Exclusion criteria: hepatic encephalopathy, ascites or pregnancy, hepatorenal syndrome or hepato-pulmonary syndrome, right side heart failure (liver congestion), extra hepatic tumors, history of liver transplantation, cholestasis, and hepatitis B patients

An informed written consent was obtained for each involved patients for approval to participate in this study. All patients were subjected to the following: full history taking and clinical examination including information on age, sex, gastrointestinal symptoms, comorbidities (diabetes mellitus (DM), hypertension (HTN), and congestive heart failure (CHF)), hepatic encephalopathy, renal impairment, liver transplantation, tumors, and medications.

Blood samples were collected from the patients by vacuum venipuncture, using a dry sterile $5-\mathrm{ml}$ tube. The serum was separated, centrifuged, and aliquoted. Laboratory investigations were done for all the patients including the following: liver function tests as liver enzymes (ALT-AST), serum albumin, bilirubin, prothrombin time and INR, viral hepatitis markers (HBs Ag, anti-HBc, and anti-HCV), complete blood picture and erythrocyte sedimentation rate (ESR), kidney function tests (blood urea and serum creatinine), and serum $\alpha$-fetoprotein (AFP).

Imaging was performed for all the patients including the following: (a) abdominal ultrasonography, reporting data for liver echogencity, vascularity (portal and hepatic veins), intrahepatic biliary dilation, and focal lesion criteria (site, number, size, and echogencity); also report about the spleen, kidneys, lymph nodes, ascites, and any other abnormality if present. (b) Contrast-enhanced triphasic computerized tomography (CT) confirming HCC diagnosis. (c) Fibroscan on the liver: liver stiffness measurement (LSM) using Fibroscan 402 (Echosens, Paris, France) was done.

Principle of technique: it measures liver stiffness as it is a rapid technique taking less than $5 \mathrm{~min}$ that can be easily performed at bedside or in outpatient clinic. It is a noninvasive and painless technique, with results immediately available and it can be safely repeated for follow-up. The results are expressed in kilopascals $(\mathrm{kPa})$, corresponding to the median value of 10 validated measurements according to the manufacturer's recommendations and the machine scale result ranges from 2.5 to $75 \mathrm{kPa}$ with normal value around $5.5 \mathrm{kPa}$. The validity results also depend on two important parameters: the interquartile range, which reflects the variability of the validated measures and should not exceed $30 \%$ of the median value; the success rate (the ratio of the number of successful measurements to the total number of acquisitions) should be at least $60 \%$.

Technique: the patient was instructed to lie supine. An ultrasound-like probe was placed on the skin over the liver area, typical in the right mid axillary line. The patient felt a gentle flick each time a vibration wave is generated by the probe. Then the patients were evaluated practically according to the following scores:

Modified Child score: evaluation of the severity of liver cirrhosis was obtained in each cirrhotic patient with modified Child-Pugh score. This system relies on clinical and laboratory evaluation including ascites, grade of encephalopathy, serum albumin, bilirubin, and prothrombin time.

Model of End-stage Liver Disease (MELD) score: this is a model for end-stage liver disease for evaluation of the severity of liver cirrhosis in each cirrhotic patient, and this system relies on laboratory evaluation including serum bilirubin, serum creatinine, and INR (international normalized ratio). MELD score $=9.6 \times \log ($ creatinine $\mathrm{mg} / \mathrm{dl})+3.8 \times \log ($ bilirubin $\mathrm{mg} / \mathrm{dl})+11.2 \times \log$ (INR) + 6.4.

Statistical analysis: statistical presentation and analysis of the present study was conducted, using the mean, standard deviation, and chi-square test by SPSS V.16. Chi-square test was used for comparison between two groups as regards qualitative data. Significance level $(P)$ value was expressed as follows: $P$ value $\geq 0.05$ was considered statistically non-significant; $P$ value $<0.05$ was considered statistically significant; $P$ value $<0.001$ was considered statistically highly significant. 


\section{Results}

This study included fifty HCV cirrhotic patients who were divided into two groups: group I, included $25 \mathrm{HCV}$ cirrhotic patients (16 male and 9 female) with hepatocellular carcinoma and group II, included $25 \mathrm{HCV}$ cirrhotic patients (12 male and 13 female) without evidence of hepatocellular carcinoma. Age and sex were cross matched between the two studied groups. The demographic data of both groups are shown in Table 1.

As regards the reported clinical data, 13 patients (52\%) in group I and 3 patients (12\%) in group II presented by jaundice, while 20 patients $(80 \%)$ in group I and 9 patients $(36 \%)$ in group II presented by clubbing. Nineteen patients $(76 \%)$ in group I and 10 patients $(40 \%)$ in group II presented by ecchymosis, and hepatomegaly was detected in 2 patients (8\%) in group I and 6 patients $(24 \%)$ in group II; feeling of hard liver was detected in 10 HCC patients (40\%) compared to HCV cirrhotic patients without HCC with a significant statistical difference. Splenomegaly was detected in 18 patients $(72 \%)$ in group I and 20 patients(80\%) in group II, while 15 patients (60\%) in group I and 10 patients $(40 \%)$ in group II suffered from abdominal pain; while 20 patients (80\%) in group I and 16 patients (64\%) in group II suffered from easy fatigability, 22 patients $(88 \%)$ in group I and 20 patients (80\%) in group II suffered from loss of weight. Jaundice, clubbing, and ecchymosis were more in HCC patients than in cirrhotic patients with a significant statistical difference. No patients had ascites or hepatic encephalopathy. Comparison between both groups according to clinical data is shown in Table 2 .

Table 1 Comparison between the two studied groups according to their demographic data

\begin{tabular}{|c|c|c|c|c|c|}
\hline & \multicolumn{2}{|c|}{ Group I $(\boldsymbol{n}=25)$} & \multicolumn{2}{|c|}{ Group II $(\boldsymbol{n}=25)$} & \multirow[t]{2}{*}{$P$} \\
\hline & No. & $\%$ & No. & $\%$ & \\
\hline \multicolumn{6}{|l|}{ Sex } \\
\hline Male & 16 & 64.0 & 12 & 48.0 & \multirow[t]{2}{*}{0.254} \\
\hline Female & 9 & 36.0 & 13 & 52.0 & \\
\hline \multicolumn{6}{|l|}{ Age (years) } \\
\hline Min.-Max. & \multicolumn{2}{|c|}{$40.0-62.0$} & \multicolumn{2}{|c|}{$37.0-50.0$} & \multirow[t]{3}{*}{0.918} \\
\hline Mean \pm SD & \multicolumn{2}{|c|}{$56.36 \pm 4.83$} & \multicolumn{2}{|c|}{$46.20 \pm 6.01$} & \\
\hline Median & \multicolumn{2}{|l|}{50.0} & \multicolumn{2}{|c|}{45.0} & \\
\hline \multicolumn{6}{|l|}{ Occupation } \\
\hline Farmer & 14 & 56.0 & 11 & 44.0 & \multirow[t]{2}{*}{0.396} \\
\hline Non-farmer & 11 & 44.0 & 14 & 56.0 & \\
\hline \multicolumn{6}{|l|}{ BMI $\left(k g / m^{2}\right)$} \\
\hline Min.-Max. & \multicolumn{2}{|c|}{$22.0-26.0$} & \multicolumn{2}{|c|}{$20.98-25.23$} & $0.018^{*}$ \\
\hline Mean \pm SD & \multicolumn{2}{|c|}{$24.49 \pm 1.0$} & \multicolumn{2}{|c|}{$23.66 \pm 1.39$} & \\
\hline Median & \multicolumn{2}{|c|}{24.60} & \multicolumn{2}{|c|}{24.11} & \\
\hline
\end{tabular}

Comparison between the two studied groups according to median and IQR of Fibroscan is shown in Table 3.

As regards binary logistic regression for predictors of HCC, it was found that Child C, AST, Fibroscan, and AFP were predictors for developing $\mathrm{HCC}$. The highest odds ratio was Child $\mathrm{C}(P$ 0.035) followed by $\mathrm{AFP}(P$ 0.058) then Fibroscan $(P$ 0.88) and AST ( $P$ 0.76). This is well demonstrated in Table 4.

As regards AFP values, values were higher in HCC patients than in cirrhotic patients $(P=0.003)$, with a significant difference between the studied groups. Liver stiffness values were significantly high in all groups, and the specific cutoff value for hepatocellular carcinoma detection was above $24 \mathrm{kPa}$ in hepatitis $\mathrm{C}$ virus patients. Therefore, liver stiffness of more than $24 \mathrm{kPa}$ can be considered as an independent risk factor for the development of liver cancer in HCV patients. The cutoff values for Fibroscan and AFP as markers for HCC are shown in Table 5.

\section{Discussion}

The presence of cirrhosis represents a key risk factor for the development of HCC. The HCC incidence rate of cirrhosis is $2-4 \%$ per year, so patients with cirrhosis constitute a high risk group for effort at prevention and early detection [19].

So in this study, we aimed to determined role and clinical significance of Fibroscan for early detection of HCC in hepatitis $\mathrm{C}$ Cirrhotic patients. In order to achieve this goal, we selected fifty subjects who were divided into two groups: group I included $25 \mathrm{HCV}$ cirrhotic patients (16 male and 9 female) with hepatocellular carcinoma, while group II included $25 \mathrm{HCV}$ cirrhotic patients (12 male and 13 female) without evidence of HCC.

In the current study, AFP in cases of liver cancer (HCC) had an average value of $317.5 \mathrm{ng} / \mathrm{ml}$ which was statistically higher than patients with cirrhosis $(10.8 \mathrm{ng} /$ $\mathrm{ml})$. These results are consistent with Jiang et al. [20] who reported that AFP in cases of liver cancer had an average value of $384.6 \mathrm{ng} / \mathrm{ml}$ which was statistically higher than patients with cirrhosis $(26.04 \mathrm{ng} / \mathrm{ml})$.

On the other hand, other studies have shown that the role of AFP in diagnosing liver cancer is limited, and these results were supported by El-Serag et al. [21] who stated that AFP was not elevated in all patients with liver cancer. Its sensitivity to detect liver cancer is $79 \%$; the specificity is also $89 \%$ and not $100 \%$ because AFP in the serum can also be detected in patients with cirrhosis and chronic hepatitis. This was supported by Abdel-Rahman et al. [22] who stated that the AFP level is always normal in patients with small HCC and does not rise significantly in patients with early stage, and curable liver cancer may be offset. Huaibin et al. [23] also concluded that 
Table 2 Comparison between the studied groups according to clinical data

\begin{tabular}{|c|c|c|c|c|c|}
\hline \multirow[t]{2}{*}{ Clinical data } & \multicolumn{2}{|c|}{ Group (I) HCC $\boldsymbol{n}=25$} & \multicolumn{2}{|c|}{ Group (II) cirrhosis $\boldsymbol{n}=25$} & \multirow[t]{2}{*}{$\boldsymbol{P}$ value } \\
\hline & $n$ & $\%$ & $n$ & $\%$ & \\
\hline Jaundice & 13 & 52 & 3 & 12 & $0.03 *$ \\
\hline Clubbing & 20 & 80 & 9 & 36 & $<0.001^{*}$ \\
\hline Ecchymosis & 19 & 76 & 10 & 40 & $0.02^{*}$ \\
\hline Hepatomegaly & 2 & 8 & 6 & 24 & 0.5 \\
\hline Hard liver & 10 & 40 & 0 & 0 & $0.004^{*}$ \\
\hline Splenomegaly & 18 & 72 & 20 & 80 & 0.13 \\
\hline Abdominal pain & 15 & 60 & 10 & 40 & 0.903 \\
\hline Easy fatigability & 20 & 80 & 16 & 64 & 0.65 \\
\hline Loss of weight & 22 & 88 & 20 & 80 & 0.865 \\
\hline
\end{tabular}

*Significant

the AFP level in the blood was a poor diagnostic indicator in liver cancer patients.

In our study, the ROC analysis of AFP when used as a diagnostic test indicates that a value of around $26.15 \mathrm{ng} /$ $\mathrm{ml}$ provides the optimum balance between sensitivity and specificity, but at this level the sensitivity is only $79 \%$ and specificity is $89.5 \%$. This is consistent with Jiang et al. [20] who reported that the sensitivity of AFP was around $79.7 \%$ and with specificity of about $80.3 \%$ in cases of liver cancer.

So as a need of new screening tools, this work studied Fibroscan as a noninvasive method for measuring liver stiffness as a risk and diagnostic predictors for HCC.

Our data reported that TE may be a useful and promising noninvasive method for liver fibrosis assessment and it is a good diagnostic predictor for HCC development in HCV cirrhotic patients.

The present study reported cutoff value of $24 \mathrm{kPa}$ for diagnostic prediction of HCC with sensitivity $100 \%$, specificity 83.3\%, PPV 94.5\%, NPV 77.3\%, and AUC 89\%.

Singh et al. [24] reported that the degree of liver stiffness was associated with risk of decompensated cirrhosis, HCC,

Table 3 Comparison between the two studied groups according to median and IQR of Fibroscan

\begin{tabular}{|c|c|c|c|c|}
\hline & Group I $(\boldsymbol{n}=25)$ & Group II $(\boldsymbol{n}=25)$ & $T$ & $P$ \\
\hline \multicolumn{5}{|c|}{ Median of Fibroscan } \\
\hline Min.-Max. & $18.0-68.5$ & $14.0-36.0$ & $18.086^{*}$ & $<0.003^{*}$ \\
\hline Mean \pm SD & $42.08 \pm 6.92$ & $25.2 \pm 3.0$ & & \\
\hline Median & 40.0 & 24 & & \\
\hline \multicolumn{5}{|c|}{ IQR of Fibroscan } \\
\hline Min.-Max. & $4.0-16.0$ & $1.80-11.60$ & $16.723^{*}$ & $<0.003^{*}$ \\
\hline Mean \pm SD & $9.08 \pm 0.81$ & $5.21 \pm 0.27$ & & \\
\hline Median & 8.0 & 4.8 & & \\
\hline
\end{tabular}

and death in patients with CLDs. LSM therefore might be used in risk stratification.

Tatsumi et al. [2] reported liver stiffness measurement in their work for risk assessment of hepatocellular carcinoma and found that in HCV, liver stiffness of more than $12.0 \mathrm{kPa}$ was an independent risk factor for new HCC development. Collectively, determining the fibrotic cutoff values for HCC concurrence would be important in evaluating $\mathrm{HCC}$ risks.

Staging hepatitis $C$ in seven categories using transient elastography (Fibroscan) was determined by Poynard et al. [25] who suggested that TE increase was associated with the occurrence of all severe complications including hepatocellular carcinoma, hepatic insufficiency.

Also, in the study of Adler et al. [17] which was a retrospective observational study shows an association between liver stiffness measurements at the upper extreme and increasing $\mathrm{HCC}$ risk in patients with cirrhosis. LSM by Fibroscan allows stratification of HCC risk in a noninvasive and reliable way [26-30].

The limitation of the study was that it was centered at the hepatitis $\mathrm{C}$ cirrhosis patients. We will consider application of this idea in hepatitis B cirrhosis and other etiologies of chronic liver disease in future research.

Table 4 Binary logistic regression for significant risk factors or predictors of HCC

\begin{tabular}{llllll}
\hline Parameter & $\boldsymbol{X}$ regression & $\boldsymbol{P}$ & Odds ratio & Lower & Upper \\
\hline Child C & 3.08 & 0.035 & 7.08 & 1.02 & 68.62 \\
AST & 0.003 & 0.765 & 0.65 & 0.876 & 1.09 \\
Fibroscan & 0.001 & 0.88 & 0.76 & 0.73 & 1.01 \\
AFP & 2.22 & 0.058 & 0.83 & 0.76 & 1.05 \\
\hline
\end{tabular}


Table 5 Fibroscan and AFP cutoff values as markers for HCC

\begin{tabular}{llllllll}
\hline Test & Cutoff & Sensitivity $\%$ & Specificity $\%$ & PPV \% & NPV \% & AUC \% & $\boldsymbol{P}$ value \\
\hline Fibroscan $\mathbf{( k P a )}$ & 24 & 98.2 & 83.8 & 94.5 & 77.3 & 89 & $\mathbf{0 . 0 0 \mathbf { 0 } ^ { * }}$ \\
AFP $(\mathbf{n g} / \mathbf{m l})$ & 26.15 & 79 & 89.5 & 97.5 & 96.3 & 83.2 & $\mathbf{0 . 0 0 \mathbf { 1 } ^ { * }}$
\end{tabular}

*Significant

\section{Conclusion}

Fibroscan can be a good technique for detection of HCC high-risk cirrhotic patients and can be of great added value if incorporated in the current HCC screening protocols in hepatitis $\mathrm{C}$ cirrhotic patients. Our work is unique in selecting the studied patients of the same etiology (HCV) aiming an accurate results for this category of patient that represent the majority of HCC in Egyptian patients, while previous studies evaluated patients of different etiologies and each etiology has its own calibration on Fibroscan machine.

\section{Abbreviations}

AFP: Alfa-fetoprotein; Alb: Albumin; ALT: Alanine transaminase; AST: Aspartate transaminase; BMI: Body mass index; CBC: Complete blood count; DM: Diabetes mellitus; Hb: Hemoglobin; HBV: Hepatitis B virus; HCC: Hepatocellular carcinoma; HCV: Hepatitis C virus; HTN: Hypertension; IQR: Interquartile range; LSM: Liver stiffness measurement; No: Number; PCR: Polymerase chain reaction; PLT: Platelets; ROC: Receiving operator curve; SD: Standard deviation; SVR: Sustained virological response; USA: United States of America; WBCs: White blood cells

\section{Acknowledgements}

The authors acknowledge all patients participated in this study and take responsibility for all aspects of the reliability and freedom from bias of the data presented and their discussed interpretation.

\section{Authors' contributions}

$A E, M S$, and $S A$ were responsible for the concept, design, definition of intellectual content, and manuscript preparation. AE, MS, SA, and SA-E were responsible for literature search, manuscript review, and manuscript editing. $A E, M S, M H, M Y, S A$, and SA-E were responsible for data acquisition and clinical studies. MH and SA-E were responsible for data analysis and statistical analysis. All authors have been read and approved the final version of the manuscript.

\section{Funding}

This research received no specific grant from any funding agency in the public, commercial, or not-for-profit sectors.

\section{Availability of data and materials}

The datasets used and/or analyzed during the current study are available from the corresponding author on reasonable request.

\section{Ethics approval and consent to participate}

The research was approved from Tanta University, Faculty of Medicine Research ethical committee. The approval number is 30679/04/16. An informed written consent was taken from each patient. The study protocol complies with the ethical guidelines of the 1975 Declaration of Helsinki as reflected in prior approval by the institution's Human Research Committee.

\section{Consent for publication}

All patients included in this research gave written informed consent to publish the data contained within this study. If the patient was less than 16 years old, deceased, or unconscious when consent for publication was requested, written informed consent for the publication of this data was given by their parent or legal guardian.

\section{Competing interests}

The authors declare that there is no conflict of interest.

\section{Author details}

${ }^{1}$ Kafr-Elsheikh General Hospital, Kafr-Elsheikh, Egypt. ²Department of Tropical Medicine, Faculty of Medicine, Tanta University Hospital, Tanta University, El Geish Street, Tanta, Gharbia Governorate, Egypt. ${ }^{3}$ Diagnostic Radiology Department, Tanta University, Tanta, Egypt.

Received: 11 February 2020 Accepted: 16 July 2020

Published online: 22 July 2020

\section{References}

1. Jemal A, Bray F, Center MM et al (2011) (2011): Global cancer statistics. CA Cancer J Clin. 61:69-90

2. Tatsumi A, Maekawa S, Sato M et al (2015) (2015): Liver stiffness measurement for risk assessment of hepatocellular carcinoma. Hepatol Res. 45(5):523-532

3. Abd-Elsalam S, Elwan N, Soliman $\mathrm{H}$ et al $(2018,2018)$ Epidemiology of liver cancer in Nile delta over a decade: a single-center study. South Asian J Cancer:24-26

4. Sheta E, El-Kalla F, El-Gharib M, et al. (2016): Comparison of single-session transarterial chemoembolization combined with microwave ablation or radiofrequency ablation in the treatment of hepatocellular carcinoma: a randomized-controlled study. Eur J Gastroenterol Hepatol. 2016 28(10):1198-1203.

5. Negm O, Abou Saif S, El Gharib M et al (2017) (2017): Role of low-molecularweight heparins in prevention of thromboembolic complication after transarterial chemoembolization in hepatocellular carcinoma. Eur J Gastroenterol Hepatol 29(3):317-321

6. Watany M, Badawi R, Elkhalawany W et al (2017) (2017): Study of Dickkopf-1 (DKK-1) gene expression in hepatocellular carcinoma patients. J Clin Diagn Res 11(2):OC32-OC34

7. Elwan N, Salem ML, Kobtan A et al (2018) (2018): High numbers of myeloid derived suppressor cells in peripheral blood and ascitic fluid of cirrhotic and HCC patients. Immunol Invest 47(2):169-180

8. Abdelfattah AAM, Rizk F, Hawash N et al (2018) (2018): Randomized trial of preoperative administration of oral pregabalin for postoperative analgesia in patients scheduled for radiofrequency ablation of focal lesions in the liver. Int J Hyperthermia 34(8):1367-1371

9. Chen K, Chang P, Goh GB, et al. (2018): Surveillance for hepatocellular carcinoma - current status and advances. Hepatoma Res 2018; 4:72

10. Zacharakis G, Aleid A, Aldossari KK (2018) (2018): New and old biomarkers of hepatocellular carcinoma. Hepatoma Res 4:65

11. Richardson P, Duan Z, Kramer J et al (2012) (2012): Determinants of serum alpha-fetoprotein levels in hepatitis C-infected patients. Clin Gastroenterol Hepatol 10(4):428-433

12. Nguyen K, Jimenez M, Moghadam N et al (2017) (2017): Decrease of alphafetoprotein in patients with cirrhosis treated with direct-acting antivirals. J Clin Transl Hepatol 5(1):43-49

13. Chin JL, Pavlides M, Moolla A et al (2016) (2016) Non-invasive markers of liver fibrosis: adjuncts or alternatives to liver biopsy? Front Pharmacol 7:159

14. Wang $Y$, Hou JL (2015) (2015): Current strategies for quantitating fibrosis in liver biopsy. Chin Med J (Engl) 128(2):252-258

15. European Association for the Study of the Liver (2018) (2018): EASL clinical practice guidelines: management of hepatocellular carcinoma. J Hepatol 69(1):182-236

16. Kim JE, Ryoo BY, Ryn MH et al (2011): Sorafenib for hepatocellular carcinoma according to Child-Pugh class of liver function. Cancer Cheomother. Pharmacol. 68:1285-1290

17. Adler M, Larocca L, Trovato FM et al (2016) (2016): Evaluating the risk of hepatocellular carcinoma in patients with prominently elevated liver stiffness measurements by FibroScan: a multicentre study. HPB (Oxford) 18(8):678-683

18. Pesce A, Scilletta R, Branca A et al (2012) (2012): Does transient elastography (FibroScan ${ }^{\oplus}$ ) have a role in decision making in hepatocellular carcinoma? HPB (Oxford) 14(6):403-408 
19. Heimbach JK, Kulik LM, Finn RS et al (2018) (2018): AASLD guidelines for the treatment of hepatocellular carcinoma. Hepatology. 67(1):358-380

20. Jiang WC, Shen Y et al (2011): Clinical application of determining serum AFP-IgM complexes for diagnosis of small hepatocellular carcinoma. Anticancer Res. 31(2):687-691

21. El-Serag HB, Marrero JA, Rudolph et al (2016) (2011): Diagnosis and treatment of hepatocellular carcinoma. Gastroenterology 134(6):1752-1763

22. Abdel-Rahman A, Alam El-Din HM, Bahnassy AA et al (2010) (2010): Serum levels of soluble Fas, soluble tumor necrosis factor-receptor II, interleukin-2 receptor,transient elastography and interleukin-8 as early predictors of hepatocellular carcinoma in Egyptian patients with hepatitis $C$ virys genotype-4.J. Hepatology 9:1

23. Huaibin M, Juan C, Hernandez et al (2012) (2012). Morphologic features of extrahepatic manifestations of hepatitis C virus infection. Clin Dev Immunol. 2012:740138

24. Singh S, Fujii L, Ehman R et al (2013) (2013): Decompansated liver cancer of chronic liver diseases. Clin Gastroenterol Hepatol 11(12):157384.e1-1573-84.e2

25. Poynard T, Vergniol J, Ngo Y et al (2014) (2014):Staging chronic hepatitis C in seven categories using fibrosis biomarker (FibroTest ${ }^{\mathrm{TM}}$ ) and transient elastography (FibroScan ${ }^{\oplus}$ ). Hepatol 60(4):706-714

26. Haydara T, Gabr M, Abofreikha M et al $(2019,2019)$ The effect of stem cell transplantation therapy for post viral chronic liver cell failure on associated type II diabetes mellitus: a pilot study. Endocr Metab Immune Disord Drug Targets. https://doi.org/10.2174/1871530319666191202125402 [Epub ahead of print]

27. Soliman H, Ziada D, Salama M et al (2020) (2020): Predictors for fibrosis regression in chronic HCV patients after the treatment with DAAS: results of a real-world cohort study. Endocr Metab Immune Disord Drug Targets. 20(1):104-111

28. Hanafy AS, Seleem WM, El-Kalla F et al (2019) (2019): Efficacy of a noninvasive model in predicting the cardiovascular morbidity and histological severity in non-alcoholic fatty liver disease. Diabetes Metab Syndr 13(3): 2272-2278

29. Abd-Elsalam S, Habba E, Elkhalawany W et al (2016) (2016): Correlation of platelets count with endoscopic findings in a cohort of Egyptian patients with liver cirrhosis. Medicine (Baltimore) 95(23):e3853

30. Rizk FH, Sarhan NI, Soliman NA et al (2018) (2018): Heat shock protein 47 as indispensible participant in liver fibrosis: possible protective effect of lactoferrin. IUBMB Life. 70(8):795-805

\section{Publisher's Note}

Springer Nature remains neutral with regard to jurisdictional claims in published maps and institutional affiliations.

\section{Submit your manuscript to a SpringerOpen ${ }^{\circ}$ journal and benefit from:}

- Convenient online submission

- Rigorous peer review

- Open access: articles freely available online

- High visibility within the field

- Retaining the copyright to your article

Submit your next manuscript at $\boldsymbol{\nabla}$ springeropen.com 